\title{
Thermal response of nanoscale cylindrical inclusions of amorphous silica embedded in $\alpha$-quartz
}

\author{
B. Afra, ${ }^{1}$ K. Nordlund, ${ }^{2}$ M. D. Rodriguez, ${ }^{1}$ T. Bierschenk, ${ }^{1}$ C. Trautmann,${ }^{3,4}$ S. Mudie,${ }^{5}$ and P. Kluth ${ }^{1, *}$ \\ ${ }^{1}$ Department of Electronic Materials Engineering, Research School of Physics and Engineering, Australian National University, \\ Canberra ACT 2601, Australia \\ ${ }^{2}$ Department of Physics, Helsinki Institute of Physics, University of Helsinki, Helsinki, Finland \\ ${ }^{3}$ GSI Helmholtz Centre for Heavy Ion Research, Darmstadt, Germany \\ ${ }^{4}$ Technische Universität, Darmstadt, Germany \\ ${ }^{5}$ Australian Synchrotron, 800 Blackburn Road, Clayton VIC, Australia
}

(Received 9 April 2014; revised manuscript received 4 December 2014; published 30 December 2014)

\begin{abstract}
The thermal response of nanoscale cylindrical inclusions of amorphous $\mathrm{SiO}_{2}$ embedded in crystalline quartz $\left(c-\mathrm{SiO}_{2}\right)$ is investigated by means of small-angle $\mathrm{x}$-ray scattering. The inclusions are generated by swift heavy-ion irradiation that leads to the formation of amorphous ion tracks along the ion trajectories. During in situ annealing between room temperature and $620^{\circ} \mathrm{C}$, we observe an irreversible expansion of the track cylinders followed by a reversible contraction. The reversible "elastic" response of the tracks can be modeled using the temperature-dependent elastic properties of bulk amorphous and crystalline $\mathrm{SiO}_{2}$ for the inclusions and the matrix, respectively. A high initial interface pressure of the nanocylinders is apparent from the calculations.
\end{abstract}

DOI: 10.1103/PhysRevB.90.224108

PACS number(s): 61.05.cf, 61.80.Jh, 62.20.D-

\section{INTRODUCTION}

Amorphous cylindrical inclusions embedded in a crystalline matrix, such as ion tracks in $\alpha$-quartz generated by swift heavy-ion irradiation [1,2] represent a model system for nanoscale "infinite" cylindrical inclusions embedded in a matrix. Such tracks are only a few nanometers in diameter and can be up to tens of micrometers long [3-5]. The amorphous tracks are sensitive to thermal annealing and at elevated temperatures can shrink in size and eventually completely recrystallize [6-8]. We previously demonstrated that synchrotron-based small-angle x-ray scattering (SAXS) provides a powerful tool to determine track radii with angstrom precision $[6,9,10]$. The scattering is measured from $\sim 10^{7}$ ion tracks generated under identical conditions and is thus representative of the individual track structure averaging out fluctuations on an atomic level. The technique is in particular suitable for materials where the boundary of the track is well defined due to an amorphous-crystalline interface [6,11]. In combination with in situ annealing experiments, it is possible to not only study kinetic processes during recrystallization, but also study changes that may occur as a result of the elastic behavior of the tracks. This paper focuses on the thermal response of ion tracks in $\alpha$-quartz in the temperature range of up to $620^{\circ} \mathrm{C}$. We observe a peculiar change in the radius of these nanoscale amorphous regions before the beginning of track recrystallization. This can be well explained by a drastic change in the Poisson ratio of quartz near the $\alpha$ to $\beta$ transition temperature using continuum elasticity theory. The measurements combined with a model calculation enable us to estimate the pressure that the tracks are exposed to prior to annealing. The study demonstrates both the applicability of the theory at the nanoscale and the ability to reliably measure the resulting changes using SAXS.

${ }^{*}$ Corresponding author: patrick.kluth@anu.edu.au

\section{EXPERIMENT}

Ion tracks representing elastic inclusions were generated in single-crystalline $360-\mu \mathrm{m}$ thick wafers of synthetic $\alpha$-quartz using $2.0-\mathrm{GeV}^{238} \mathrm{U}$ ions at the UNILAC accelerator at GSI (Darmstadt, Germany). The irradiations were performed at room temperature, under normal beam incidence, and with fluences of $0.5,1$, and $3 \times 10^{11} \mathrm{ions} / \mathrm{cm}^{2}$. According to SRIM 2008 calculations, the projected range of the ions is $\sim 70 \mu \mathrm{m}$ [12]. It is apparent that the ion tracks generate high stresses as samples exposed to higher fluences easily fracture when handled. After irradiation, the samples were mechanically polished from the backside using a tripod method. Samples irradiated with 0.5 and $1 \times 10^{11}$ ions $/ \mathrm{cm}^{2}$ were thinned down to a thickness between 50 and $90 \mu \mathrm{m}$ whereas samples irradiated with $3 \times 10^{11}$ ions $/ \mathrm{cm}^{2}$ fractured during polishing and thus were only polished to approximately $240 \mu \mathrm{m}$.

SAXS was combined with in situ annealing experiments in order to study the temperature dependence of the ion track radii. Transmission SAXS measurements were conducted at the Australian Synchrotron with an x-ray energy of $12 \mathrm{keV}$ and a camera length of approximately $1600 \mathrm{~mm}$. The experimental setup is schematically shown in Fig. 1(a) including a threeaxis goniometer stage with a heating facility. Annealing was performed using a LINKAM TS1500 heating stage positioned normal to the x-ray beam. The goniometer allowed for precise tilting of the sample in front of the beam. The temperature was stepwise increased from room temperature up to a maximum of $620^{\circ} \mathrm{C}$ with a temperature profile illustrated in Fig. 1(c). The ramping rate between different temperature steps was $130^{\circ} \mathrm{C} / \mathrm{min}$, and the dwell time at each temperature was $5 \mathrm{~min}$. After $5 \mathrm{~min}$ at $620^{\circ} \mathrm{C}$, the sample was cooled down to $50{ }^{\circ} \mathrm{C}$ and was kept at this temperature for $15 \mathrm{~min}$. This cycle of annealing was repeated for a second time (for some samples also for a third time). During the annealing process and the cooling period, SAXS measurements were taken periodically with 6 - and 15-s exposure times followed by approximately $40 \mathrm{~s}$ of delay. 

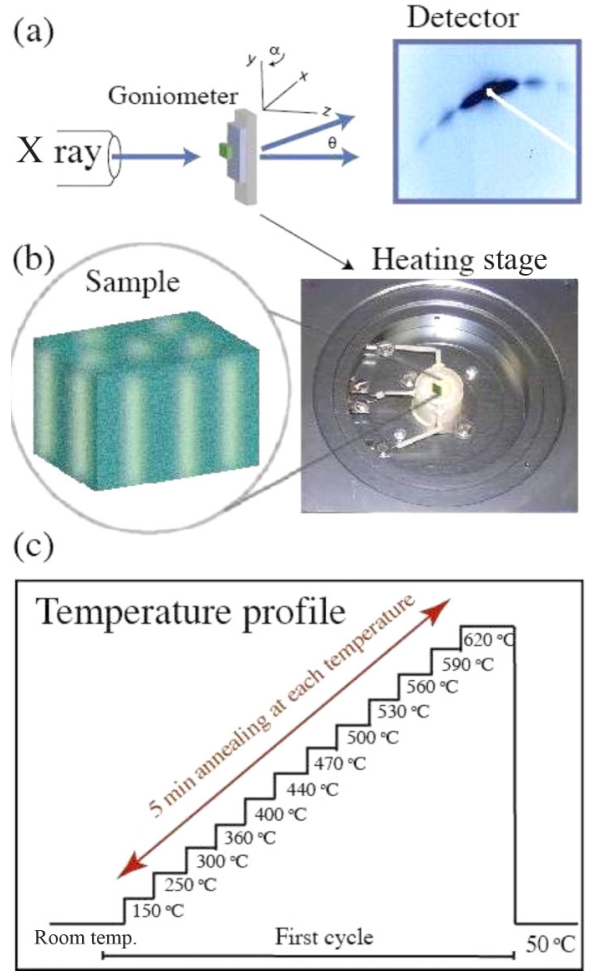

FIG. 1. (Color online) (a) Scheme of the experimental setup for SAXS measurements combined with in situ annealing. (b) The heating stage for in situ annealing. A schematic of a sample containing tracks is also shown (the scale is not representative of the real ion tracks). (c) Temperature profile during in situ annealing experiments.

\section{RESULTS AND DISCUSSION}

\section{A. SAXS measurements}

Figure 2 shows SAXS images from an irradiated sample at room temperature and after 5-min annealing at $500^{\circ} \mathrm{C}$. The track axis was tilted by $\sim 10^{\circ}$ with respect to the $\mathrm{x}$-ray beam. The curved streaks with oscillating intensities are indications of well parallel oriented continuous cylindrical regions with a different electron density than the surrounding matrix [6,11]. The shape of this signal is a consequence of the high aspect ratio of the cylindrical

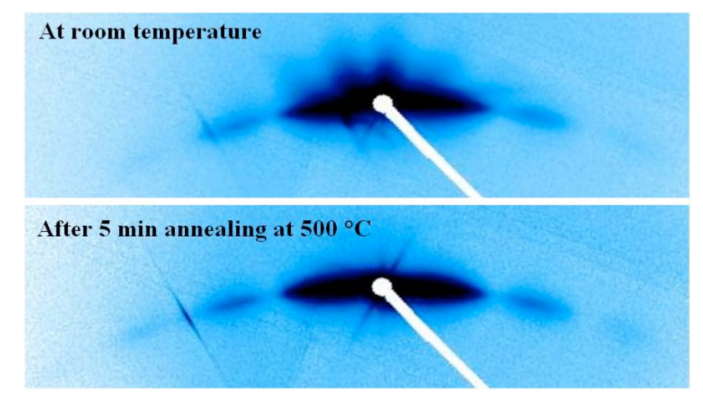

FIG. 2. (Color online) SAXS images of a quartz sample containing tracks produced with $2.0-\mathrm{GeV} \mathrm{U}$ ions; (top) at room temperature and (bottom) when annealed at $500{ }^{\circ} \mathrm{C}$ after having passed a temperature profile with 5-min annealing steps at different temperature steps. The axis of the tracks is tilted by $\sim 10^{\circ}$ with respect to the x-ray beam.

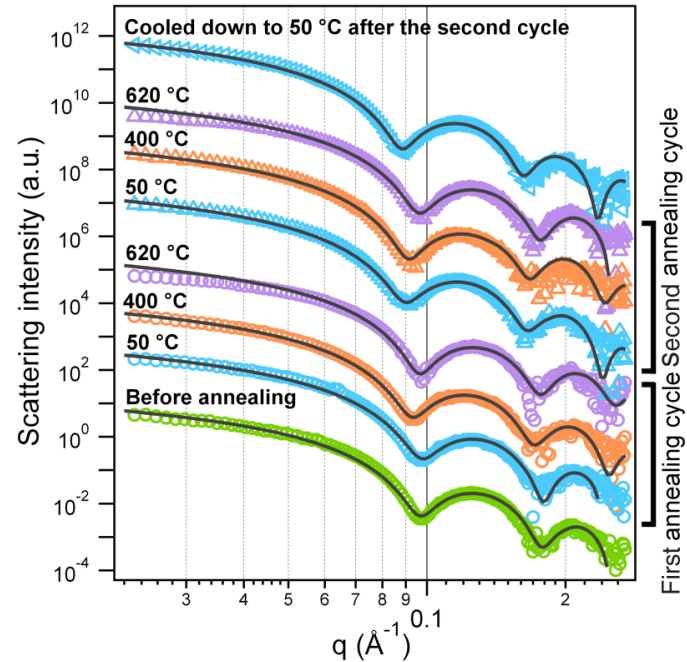

FIG. 3. (Color online) SAXS spectra from the amorphous cylindrical inclusions in quartz generated with $2.0-\mathrm{GeV} \mathrm{U}$ ions to a fluence of $1 \times 10^{11}$ ions $/ \mathrm{cm}^{2}$. SAXS data were recorded at room temperature before annealing and at different temperatures between 50 and $620^{\circ} \mathrm{C}$ for two consecutive cycles. Solid lines are fits to the hard-cylinder model [11]. The spectra are offset for clarity.

tracks that are only few nanometers in diameter and tens of micrometers long [11]. The intensity along the streaks contains information about the radial density profile of the cylinders.

From the streaky patterns, the scattering intensities as a function of the scattering vector are extracted and after background subtraction analyzed. Figure 3 shows the SAXS data for several selected temperatures during the first and the second annealing cycles at the end of the 5-min annealing steps. The shape, i.e., the undulating character of the scattering intensities does not change with temperature, indicating that the cylindrical track geometry is maintained. The small shift in the minima gives clear evidence of a change in track radii [6]. The SAXS spectra were best fitted using a cylindrical model that assumes a sharp transition of the electron density between the track and the surrounding matrix (hard cylinder). This is consistent with previous SAXS measurements [11] and the formation of amorphous tracks embedded in the crystalline quartz matrix. The solid lines in Fig. 3 represent the fits using the hard-cylinder model [11].

Figure 4 shows the extracted cylinder radii as a function of the annealing temperature during (a) the first and (b) second and third annealing cycles (symbols). The radii correspond to data recorded after $5 \mathrm{~min}$ annealing at a given temperature. In the first cycle the evolution of the radius is characterized by an initial increase up to about $400^{\circ} \mathrm{C}$ followed by a sharp decrease. Upon returning to $\sim 50^{\circ} \mathrm{C}$, before the second cycle started, the radius was found to be significantly larger than the initial value (before annealing), indicating an irreversible expansion of the cylindrical inclusion. The second and third annealing cycles are characterized by a sharp decrease in the radius above $\sim 400{ }^{\circ} \mathrm{C}$. The radii in the two cycles are nearly identical, indicating a reversible process. The inset in Fig. 4(a) shows the change in the radius as a function of time during annealing at $250{ }^{\circ} \mathrm{C}$. Even though the total change during this 

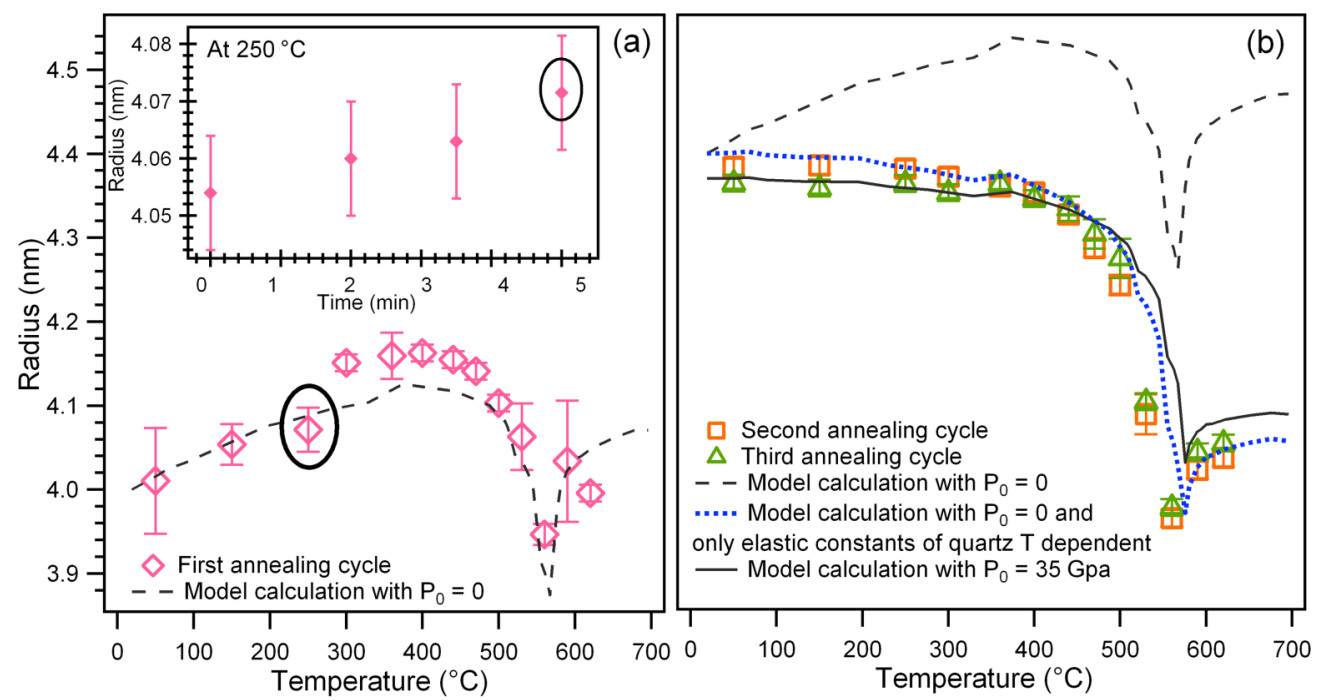

FIG. 4. (Color online) Track radii in quartz (generated with $2.0-\mathrm{GeV}$ U ions to a fluence of $5 \times 10^{10}$ ions $/ \mathrm{cm}^{2}$ ) deduced from SAXS data as a function of the annealing temperature during (a) the first and (b) second and third annealing cycles. The radius for each temperature corresponds to that after 5-min annealing. The inset in (a) shows the change in the radius during the annealing at $250^{\circ} \mathrm{C}$ as a function of time. The dashed lines in (a) and (b) represent the model calculations for a cylindrical elastic inclusion taking into account the temperature-dependent elastic constants of amorphous silica (track) and quartz (matrix), with $P_{0}=0$. The radius at the beginning of the temperature cycle is fixed by the experimental value. In (b), the solid line shows the model calculation with an initial contact pressure $P_{0}=35$ GPa on the cylindrical inclusion and the dotted blue line results from a calculation with $P_{0}=0$ and with only the elastic constants of quartz being temperature dependent.

time is very small, it is evident that at least part of the change in the track radius occurs on a time scale of minutes. For better identification, the data points corresponding to the radius after 5 min annealing at $250{ }^{\circ} \mathrm{C}$ are circled. In contrast, in the second and third annealing cycles, the entire changes in the track radii appear to be "instant," i.e., faster than the time resolution of the experiment.

Preannealing at $400{ }^{\circ} \mathrm{C}$ for 20 min performed on a second sample irradiated under identical conditions $(2.0-\mathrm{GeV} \mathrm{U}$ ions with a fluence of $5 \times 10^{10}$ ions $/ \mathrm{cm}^{2}$ ) before exposing it to the same annealing cycle as the first sample also yielded an irreversible increase in the cylinder radius. The following annealing cycles were similar to the second and third cycles of the first sample.

Similar annealing experiments were carried out for samples irradiated at higher fluences of 1 and $3 \times 10^{11}$ ions $/ \mathrm{cm}^{2}$ (the mean distance here is still large enough to consider them as individual scattering objects). The results are very similar indicating that the number density of the tracks has no considerable effect on the temperature evolution of the radius. We thus rule out proximity effects of adjacent cylinders on the observed evolution of the track radii.

Previous investigations have shown that tracks in quartz consist of amorphous $\mathrm{SiO}_{2}$ [1,11]. A density change of only approximately $2 \%$ [11] suggests the tracks consist of inclusions of densified silica, given $\sim 15 \%$ density difference is observed for bulk amorphous silica [13].

\section{B. Modeling using continuum elasticity theory}

The observed reversible change in the track radii can be explained using the temperature-dependent elastic constants of amorphous silica $\left(a-\mathrm{SiO}_{2}\right)$ and quartz $\left(c-\mathrm{SiO}_{2}\right)$. Both silica and quartz are known to show an unusual thermal dependence of their elastic properties. Silica becomes elastically harder from room temperature up to about $1100^{\circ} \mathrm{C}[14,15]$, whereas quartz becomes elastically softer. Its aggregate Poisson's ratio changes rapidly and becomes negative close to the $\beta$-quartz transition temperature $\left(\sim 573^{\circ} \mathrm{C}\right)[16]$.

To understand how the elastic constants affect the track radius, we apply classical elasticity theory [17,18], approximating the ion track as an infinitely long cylindrical elastic inclusion in an infinite elastic medium. This approximation is justified given aspect ratios of $\sim 1 / 10000$ and the low fluence irradiation where the mean distance between different tracks is large compared to the track radius. For isotropic linear elastic materials with a volume mismatch and different elastic constants in the cylinder and surrounding medium, the static elastic behavior of this system has an exact analytical solution $[19,20]$. The radial strain $\varepsilon_{r r}^{a}$ of the material in the cylindrical inclusion (relative to the equilibrium size of the same material at zero pressure) can be written as [20]

$$
\varepsilon_{r r}^{a}=-\frac{1}{E^{a}}\left[1-v^{a}-2\left(v^{a}\right)^{2}\right] P-v^{a} \xi,
$$

where the contact pressure $P$ is given by

$$
P=-\xi \frac{1+v^{a}}{\frac{1+v^{m}}{E^{m}}+\frac{1-\nu^{a}-2\left(v^{a}\right)^{2}}{E^{a}}}+P_{0} .
$$

Here $E$ is Young's modulus, and $v$ is Poisson's ratio. The superscripts $a$ and $m$ denote the properties of the amorphous cylindrical inclusions and the crystalline surrounding matrix, respectively. $P_{0}$ is introduced here as a preexisting contact pressure consistent with high stresses produced by the ion 
tracks [11]. $\xi$ is the relative linear size mismatch between the amorphous and the matrix materials in their equilibrium states. This can be determined from the difference in the densities of the matrix and the track. In order to account for the thermal expansion of the amorphous inclusions and the matrix, we have made $\xi$ temperature dependent for both silica and quartz. For quartz, we used the thermal expansion reported in Ref. [21], which is valid in the entire temperature region and agrees well with a more recent analysis of the expansion in a narrower temperature region [22]. To be consistent with the experimental geometry, we used the $a$ - and $b$-axis values for the expansion (which are identical). For the expansion of silica, we used the values measured in a wide range of temperatures $[23,24]$ which show that the expansion in the temperature range of interest here is very close to linear, much smaller than that in quartz, and described well by an expansion coefficient of $5.1 \times 10^{-7} \mathrm{l} / \mathrm{K}$. In the current case, it is naturally uncertain whether this macroscopic thermal expansion value for silica is accurate for the nanosized ion track cylinder, but any other reasonable value much smaller than that of quartz would give essentially identical results in the current context. The linear thermal expansions for quartz and silica can be written as $\mathrm{LTE}_{\text {quartz }}=1+\alpha_{11}^{(1)} \Delta T+\alpha_{11}^{(2)} \Delta T^{2}+\alpha_{11}^{(3)} \Delta T^{3}$ [21] and $\mathrm{LTE}_{\text {silica }}=1+\alpha \Delta T$ [23], where $\Delta T=T-25^{\circ} \mathrm{C}$ and $\alpha_{11}^{(1)}=\alpha_{22}^{(1)}=13.71 \times 10^{-6}, \alpha_{11}^{(2)}=\alpha_{22}^{(2)}=6.5 \times 10^{-9}, \alpha_{11}^{(3)}=$ $\alpha_{22}^{(3)}=-1.9 \times 10^{-12}$, and $\alpha=5.1 \times 10^{-7}{ }^{\circ} \mathrm{C}^{-1}$.

Using a density $\rho_{m}=2.641 \mathrm{~g} / \mathrm{cm}^{3}$ for $\alpha$-quartz [16] and $2 \%$ lower density of the material inside the track [11] and considering that the linear size is proportional to $\rho^{-1 / 3}$, we obtain

$$
\xi=\left(\frac{2.588 \times \mathrm{LTE}_{\text {quartz }}^{3}}{2.641 \times \mathrm{LTE}_{\text {silica }}^{3}}\right)^{-1 / 3}
$$

We note that the exact value for the density of the track material is not crucial and values calculated from density differences between $0 \%$ and $15 \%$, the latter corresponding to the density difference between bulk $a-\mathrm{SiO}_{2}$ and quartz, have only an insignificant effect on the results. For the temperatures above $573{ }^{\circ} \mathrm{C}$, we have used $\rho_{m}=2.523 \mathrm{~g} / \mathrm{cm}^{3}$ for $\beta$-quartz [16].

To determine the temperature dependence of the ion track radius, we use experimental values for the temperature dependence of the elastic constants of fused silica [14] and quartz [16]. For silica we used a parabolic fit to the data reported in Ref. [14] to obtain $E^{a}$ and $v^{a}$. The selection of Young's modulus for quartz is somewhat ambiguous since a crystalline material does not have a single value for Young's modulus [17]. We used the shear modulus $C_{\mathrm{S} 1}$ for directions perpendicular to the $c$ axis. The elastic constants used are shown in Fig. 5. Other moduli [16] that are comparable in magnitude to macroscopically reported Young's moduli for commercial quartz [25] ( $70 \mathrm{GPa})$ gave similar results. For $v^{m}$ we used the aggregate Poisson's ratio $v^{*}[16]$. The radial strain $\varepsilon_{r r}^{a}$ can be calculated from the temperature-dependent elastic behavior using Eq. (1). Using the $R_{0}$ and $\varepsilon_{r r}^{a}\left(T_{0}\right)$ at room temperature, the temperature-dependent radius is given

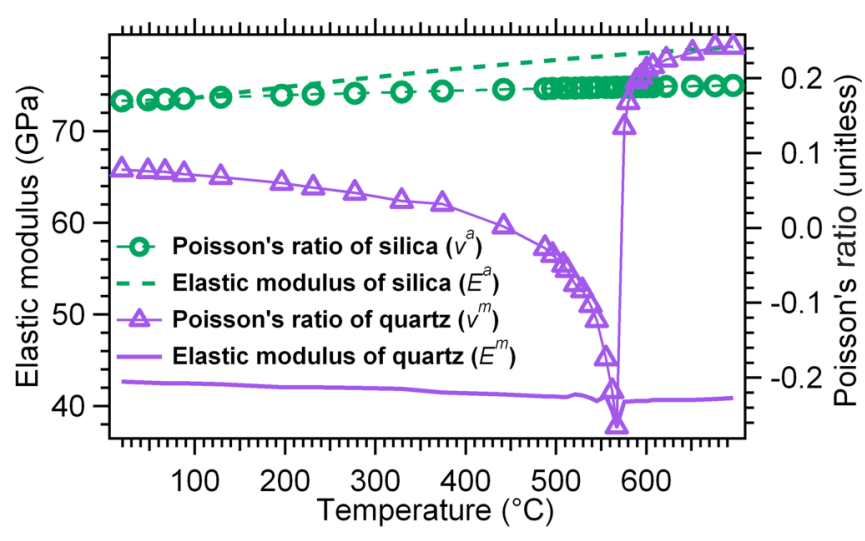

FIG. 5. (Color online) Experimental data for elastic modulus $E$ and Poisson's ratio $v$ in silica [14] and quartz [16] as a function of temperature.

by

$$
R(T)=R_{0} \frac{\left[1-\varepsilon_{r r}^{a}(T)\right]}{\left[1-\varepsilon_{r r}^{a}\left(T_{0}\right)\right]} .
$$

Calculations using this model are shown as solid and dashed lines in Fig. 4. The dashed line represents the calculation for $P_{0}=0$ and agrees well with the first annealing cycle [Fig. 4(a)]. The time dependence of the radius increase in the first annealing cycle indicates that this part is not entirely elastic in origin and the situation is more complex than the analytical model assumptions at temperatures $<400^{\circ} \mathrm{C}$. However, the sharp drop in the radius above $400^{\circ} \mathrm{C}$, which is reproduced in very similar form in the second and third annealing cycles [Fig. 4(b)], is reversible and elastic, justifying the use of the analytical model in this regime. Comparison of the analytical model results with and without the thermal expansion showed that the change in the radius is dominated by the changes in the elastic constants.

The solid line in Fig. 4(b) shows the model calculation with an initial contact pressure $P_{0}=35 \pm 3 \mathrm{GPa}$. This pressure is in the same order of magnitude as that experienced by the track at $\sim 400{ }^{\circ} \mathrm{C}$ [i.e., $P$ calculated from Eq. (2) at $400{ }^{\circ} \mathrm{C}$ with $P_{0}=0$ ]. The model yields excellent agreement with the temperature cycles following the initial irreversible increase in the track radius. The initial pressure of $35 \mathrm{GPa}$ that yields the agreement of the model with the experimental data in the second and following annealing cycles is indeed consistent with the experimentally reported transition pressure of quartz to amorphous silica at 25-35 GPa [26]. Further analysis of the atom-level stresses from a molecular-dynamics simulation performed previously [27] also indicates that the average lateral atom-level stress in the amorphous region is initially $\sim 2 \mathrm{GPa}$, corresponding well to the model with $P_{0}=0 \mathrm{GPa}$ (using $P_{0}=2 \mathrm{GPa}$ gives very similar result). These observations offer a possible explanation for the increase in the track radius. Initially the track is in a nonequilibrium state with defective dangling and stretched bonds and a low internal pressure state. The first annealing cycle relaxes the silica material towards the equilibrium state, evident in the time-dependence radius change in the Fig. 4(a) inset. Since 
normal silica is less dense than quartz, the relaxation puts the material under the compressive pressure. This pressure induces the crystalline-to-amorphous phase transition of the nearby defect-rich quartz [26], resulting in the increase of the track radius from 4.0 to $4.4 \mathrm{~nm}$ after the first cycle.

A strain in the vicinity of ion tracks in quartz has been reported previously [28], and fracturing of the samples irradiated with a fluence of $4 \times 10^{11}$ ions $/ \mathrm{cm}^{2}$ [11] confirms the high stresses in the material. As apparent from Fig. 4, if the elastic constants of the amorphous inclusions are considered independent of temperature with $P_{0}=0$, a good agreement with the reversible radius evolution is also achieved. Although there is no apparent physical justification for this scenario, it has to be considered as a possibility.

We also note that the initial irreversible expansion of the cylinders may be related to the complex volume change in silica under different temperature and pressure conditions that has been reported previously [29,30]. The volume expansion of densified silica glass annealed at different temperatures has been observed within a few minutes, and it was indicated that the annealing process is dependent on the mechanical and thermal histories [29].

In all cases, however, the dramatic drop close to the transition temperature is a result of the rapidly changing Poisson ratio of quartz close to this temperature as a negative Poisson ratio implies that the material expands sidewards on tension. In the first annealing cycle, the quartz close to the track cannot expand sidewards, hence when $v^{m}$ becomes negative, the quartz "presses back" on the silica inclusion, reducing its expansion.
Even though the model calculation indicates an increase in the track radius above the transition temperature and agrees with the available two data points, a complete understanding for the change at higher temperatures requires further measurements, and is beyond the scope of this paper.

\section{CONCLUSION}

In conclusion, we have measured an irreversible thermal expansion of radiation-induced nanoscale amorphous cylindrical inclusions embedded in $\alpha$-quartz using SAXS combined with in situ annealing. The radii of the cylinders increase irreversibly by approximately $5 \%$ up to the temperature of $\sim 400{ }^{\circ} \mathrm{C}$. Above this temperature, the amorphous inclusions undergo a reversible elastic contraction due to higher pressure exerted from the surrounding quartz crystal. The latter process is operational up to $620^{\circ} \mathrm{C}$, much before the re-crystallization of the amorphous damage begins. The pressure experienced by the cylindrical inclusions at different annealing stages has been estimated. The high accuracy of the SAXS technique to measure the cylinder radii demonstrated here offers the unprecedented ability to study the elastic behavior of suitable nanoscale systems.

\section{ACKNOWLEDGMENTS}

This research was undertaken on the SAXS/WAXS beamline at the Australian Synchrotron, Victoria, Australia. P.K. acknowledges the Australian Research Council for financial support.
[1] A. Meftah, F. Brisard, J. M. Costantini, E. Dooryhee, M. Hage-Ali, M. Hervieu, J. P. Stoquert, F. Studer, and M. Toulemonde, Phys. Rev. B 49, 12457 (1994).

[2] W. Li, L. Wang, K. Sun, M. Lang, C. Trautmann, and R. C. Ewing, Phys. Rev. B 82, 144109 (2010).

[3] M. Toulemonde, W. Assmann, C. Dufour, A. Meftah, F. Studer, and C. Trautmann, in Ion Beam Science, Solved and Unsolved Problems, edited by P. Sigmund (The Royal Danish Academy of Science and Letters, Copenhagen, 2006), pp. 263-292.

[4] C. Trautmann, in Ion Beams in Nanoscience and Technology, edited by R. Hellborg, H. J. Whitlow, and Y. Zhang, Particle Acceleration and Detection (Springer, Berlin, Heidelberg, 2010), pp. 369-387.

[5] S. Klaumuenzer, Nucl. Instrum. Methods Phys. Res., Sect. B 225, 136 (2004)

[6] B. Afra, M. Lang, M. D. Rodriguez, J. Zhang, R. Giulian, N. Kirby, R. C. Ewing, C. Trautmann, M. Toulemonde, and P. Kluth, Phys. Rev. B 83, 064116 (2011).

[7] S. Gasiorek, S. Dhar, and K. P. Lieb, Nucl. Instrum. Methods Phys. Res., Sect. B 193, 283 (2002).

[8] A. Aframian, Radiat. Eff. Defects Solids 33, 95 (1977).

[9] P. Kluth, C. Schnohr, O. H. Pakarinen, F. Djurabekova, D. Sprouster, R. Giulian, M. Ridgway, A. Byrne, C. Trautmann, D. Cookson, K. Nordlund, and M. Toulemonde, Phys. Rev. Lett. 101, 175503 (2008).
[10] B. Afra, M. D. Rodriguez, M. Lang, R. C. Ewing, N. Kirby, C. Trautmann, and P. Kluth, Nucl. Instrum. Methods Phys. Res., Sect. B 286, 243 (2011)

[11] B. Afra, M. D. Rodriguez, C. Trautmann, O. H. Pakarinen, F. Djurabekova, K. Nordlund, T. Bierschenk, R. Giulian, M. Ridgway, G. Rizza, N. Kirby, M. Toulemonde, and P. Kluth, J. Phys.: Condens. Matter 25, 045006 (2013).

[12] J. F. Ziegler, J. P. Biersack, and U. Littmark, SRIM: The Stopping and Range of Ions in Matter (Pergamon, New York, 1985).

[13] C. Trautmann, M. Boccanfuso, A. Benyagoub, S. Klaumunzer, K. Schwartz, and M. Toulemonde, Nucl. Instrum. Methods Phys. Res., Sect. B 191, 144 (2002).

[14] S. Spinner, J. Am. Ceram. Soc. 45, 394 (1962).

[15] J. A. Bucaro and H. D. Dardy, J. Appl. Phys. 45, 5324 (1974).

[16] I. Ohno, K. Harada, and C. Yoshitomi, Phys. Chem. Miner. 33, 1 (2006).

[17] J. F. Nye, Physical Properties of Crystals (Clarendon Press, Oxford, 1957).

[18] C. Kittel, Introduction to Solid State Physics, 3rd ed. (Wiley, New York, 1968).

[19] K. Nishi, A. A. Yamaguchi, J. Ahopelto, A. Usui, and H. Sakaki, J. Appl. Phys. 76, 7437 (1994).

[20] M. Grundmann, O. Stier, and D. Bimberg, Phys. Rev. B 52, 11969 (1995).

[21] R. Bechmann, A. D. Ballato, and T. J. Lukaszek, Proc. IRE 50, 1812 (1962). 
[22] J. A. Kosinski, J. G. Gualtieri, and A. Ballato, IEEE Trans. Ultrason. Ferroelectr. Freq. Control 39, 502 (1992).

[23] W. Souder and P. Hidnert, Sci. Pap. Bur. Stand. 21, 1 (1926).

[24] http://heraeus-quarzglas.com/en/quarzglas/thermalproperties/ Thermal_properties.aspx.

[25] http://www.almazoptics.com/Quartz.htm.

[26] R. J. Hemley, A. P. Jephcoat, H. K. Mao, L. C. Ming, and M. H. Manghnani, Nature (London) 334, 52 (1988).
[27] A. A. Leino, S. L. Daraszewicz, O. H. Pakarinen, F. Djurabekova, K. Nordlund, B. Afra, and P. Kluth, Nucl. Instrum. Methods Phys. Res., Sect. B 326, 289 (2014).

[28] D. M. Follstaedt, A. K. Norman, B. L. Doyle, and F. D. McDaniel, J. Appl. Phys. 100, 064306 (2006).

[29] R. Bruckner, J. Non-Cryst. Solids 5, 123 (1970).

[30] G. D. Mukherjee, S. N. Vaidya, and V. Sugandhi, Phys. Rev. Lett. 87, 195501 (2001). 